\title{
Mundhygiene durch Fachpersonal schützt Ältere vor Pneumonie
}

Hintergrund und Fragestellung: Die Healthcare-AcquiredPneumonie (HAP) ist eine häufige Erkrankung alter Menschen, sowohl im Krankenhaus als auch im Pflegeheim. Multimorbidität und Polypharmazie sind begünstigende Faktoren, aber auch die orale Hygiene scheint eine wichtige Rolle zu spielen. Mit der Analyse haben die Autoren aufgearbeitet, inwieweit assistierte Mundpflege das Mortalitätsrisiko für eine Pneumonie (hier HAP) senken kann.

Patienten und Methoden: Mit einer systematischen Literaturrecherche über PubMed, der Cochrane Libary und der Health Technology Assessment Database wurde nach randomisierten Studien gesucht, die den Effekt der intensivierten Mundpflege und den Einfluss auf die Mortalität durch HAP untersuchten. 2.678 Artikel fanden sich bei der Erstsichtung, im zweiten Durchgang wurden 2.246 Arbeiten ausgeschlossen und von den übrigen 32 Publikationen erfüllten 5 randomisierte kontrollierte Studien (RCTs) die Einschlusskriterien und wurden für die Metaanalyse herangezogen.

\section{Originalie}

Sjögren P, Wårdh I, Zimmerman $\mathrm{M}$ et al. Oral Care and Mortality in Older Adults with Pneumonia in Hospitals or Nursing Homes: Systematic Review and Meta-Analysis. J Am Geriatr Soc. 2016 Oct; $64(10): 2109-15$
Ergebnisse: Es kam heraus, dass die Mortalität durch HAP bei Patienten, deren Mundpflege von speziell zahnärztlich geschultem Personal vorgenommen worden war, deutlich gesenkt werden konnte $(\mathrm{RR}=0,43)$. Im Gegensatz dazu konnte dieser

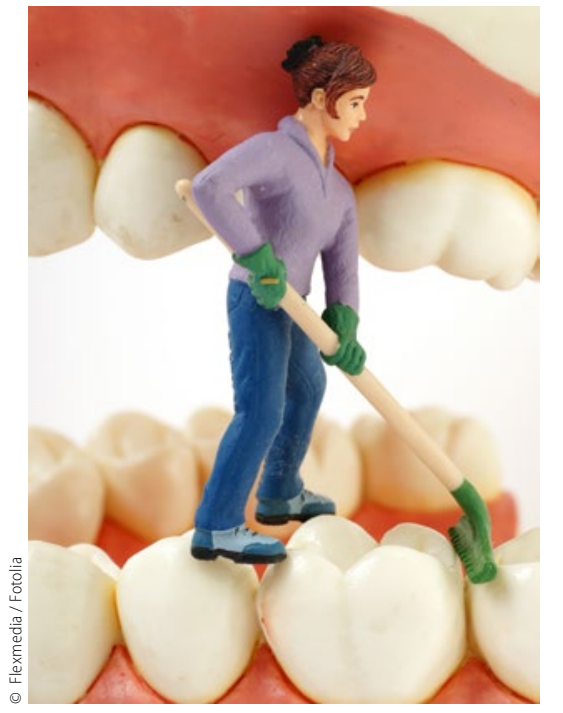

Einfach feucht durchzuwischen reicht leider nicht. Die Pflege durch zahnmedizinisch geschultes Personal bei alten Menschen bringt deutliche Vorteile.
Effekt bei der Gruppe derer, die vom regulären Personal oral gepflegt worden war, nicht nachgewiesen werden $(R R=1,20)$. Beide Pflegeverfahren wurden der sonst üblichen Pflege gegenübergestellt.

Schlussfolgerung: Die Ergebnisse zeigen, dass Mund- und Zahnpflege durch zahnmedizinisch geschultes Personal die Mortalität durch Pneumonie sowohl im Krankenhaus als auch im Pflegeheim verringern kann.

\section{- Kommentar von Univ.-Prof. Dr. med. Hans Jürgen Heppner}

\section{Wie kriegt man zahnärztlich geschultes Personal in die Routinepflege?}

Pneumonien sind nach wie vor eine der häufigsten Todesursachen bei älteren Menschen. Zu den Präventionsmaßnahmen zählen neben Impfungen, ausgewogener Ernährung und ausreichender körperlicher Aktivität auch die gute persönliche Hygiene und eine sorgfältige Mund- und Zahnpflege. So hat der Zahnstatus, mit oder ohne prothetischen Ersatz, maßgeblich Einfluss auf eine mögliche Infektion. Hinzu kommen noch Schluckstörung und Dysphagie bei multimorbiden geriatrischen Patienten [1] und eine mögliche Besiedelung durch höher pathogene Keime bei Menschen mit deutlichen Funktionseinschränkungen [2].

Zwar wird es sicherlich schwierig, zahnärztliches Fachpersonal für die Zahn- und Mundpflege in Krankenhäusern und Pflegeheimen zu gewinnen, aber es ist doch belegt, dass orale Hygiene ein bedeutender Faktor zur Verringerung von Infektionen der Atemwege bei alten Menschen ist.
Literatur

1. Komiya K, Ishii H, Kadota J. Healthcare-associated Pneumonia and Aspiration Pneumonia. Aging Dis. 2014 Feb 8;6(1):27-37

2. O Wårdh IM, Wikström MB. Long-term effects of using oral care aides at a nursing home for elderly dependent residents--a pilot study. Spec Care Dentist. 2014 Mar-Apr;34(2):64-9

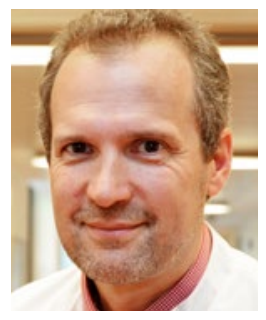

Univ.-Prof. Dr. med. Hans Jürgen Heppner

Chefarzt Geriatrische Klinik und Tagesklinik Lehrstuhl für Geriatrie Universität Witten/Herdecke Dr.-Moeller-Straße 15, 58332 Schwelm hans.heppner@uni-wh.de 